\title{
Psicooncología
}

ISSN: 1696-7240

\section{La presencia: el poder terapéutico de habitar el presente en la práctica clínica}

\author{
Enric Benito $^{1 *}$; Martín I. Mindeguía ${ }^{2}$
}

Recibido: 6 de julio de 2021 / Aceptado el 25 de agosto de 2021

Resumen. Como agentes de salud discípulos de una cosmovisión eminentemente materialista y con escasa atención a nuestro mundo interior, los profesionales sanitarios, a menudo desconocemos del potencial de nuestra presencia en el marco del encuentro terapéutico. Este artículo pretende profundizar sobre nuestra capacidad de estar presentes frente al desconocido que sufre, entendida como una condición inherente a todo ser humano y como una facultad que todos podemos reconocer, explorar y cultivar para ponerla al servicio de nosotros mismos y de aquellos a quienes acompañamos. Desde el reconocimiento del profesional como su propia herramienta terapéutica, se introducen los riesgos y oportunidades de la relación de ayuda, el rol de las emociones en el encuentro, el concepto de presencia terapéutica y el papel de la autoconciencia del profesional. Se recogen algunas referencias expertas sobre la presencia terapéutica. Se describen las bases neurofisiológicas y las características de la presencia y su papel en la co-regulación con el paciente. Y finalmente se hacen algunas propuestas para el cultivo de la presencia. Palabras clave: Presencia, presencia terapéutica, relación terapéutica, cuidados paliativos, espiritualidad, acompañamiento espiritual

\section{[en] Presence: the therapeutic power of inhabiting the present in clinical practice}

\begin{abstract}
As health agents, disciples of an eminently materialistic worldview, and with little attention to our inner world, health professionals are often unaware of their presence's potential in the framework of the therapeutic encounter. This paper aims to deepen in their ability to be present in front of an unknown suffering person, understanding this ability as an inherent condition of every human being and as a faculty that we can all recognize, explore and cultivate in order to serve ourselves and those we care for. The risks and opportunities of the helping relationship, the role of emotions in the encounter, the concept of the therapeutic presence and the role of the professional's self-awareness are introduced from the recognition of the professional as their own therapeutic tool. Some expert references on the therapeutic presence are also collected. The neurophysiological bases and the characteristics of the presence and their role on the co-regulation with the patient are described. Finally, some proposals are given for the presence cultivation.
\end{abstract}

Keywords: Presence, therapeutic presence, therapeutic relationship, palliative care, spirituality, spiritual care

1 Enric Benito. Falconer 8 bajo, 07181 Portals Nous (Calviá). Mallorca, España.

E-mail: benitoenric@gmail.com

2 Martín I. Mindeguía. C/ Mendoza 11751 piso, C1428DJE. Buenos Aires, Argentina.

E-mail: martinmindeguia@gmail.com

* Dirección de correspondencia: Enric Benito. Falconer 8 bajo, 07181 Portals Nous (Calviá). Mallorca, España. E-mail: benitoenric@gmail.com 
Sumario: 1. Introducción 2. La relación de ayuda sus riesgos y oportunidades. 3. Adentrándonos en la fisiología de las emociones y su gestión 4. La presencia como herramienta en la práctica clínica: el poder terapéutico de habitar el presente 5. ¿Que es la presencia terapéutica? 6. ¿Cuál es la base neurofisiológica de la presencia terapéutica? 7. ¿Cuáles son las cualidades características de la presencia? 8. Co-regulación: la presencia en acción 9. ¿Cómo se puede cultivar la presencia? 10. Reflexiones finales a modo de conclusión 11. Agradecimientos 12. Referencias bibliográficas.

Como citar: Benito E, Mindeguía MI. La presencia: el poder terapéutico de habitar el presente en la práctica clínica. Psicooncología 2021; 18: 371-385. https://dx.doi.org/10.5209/psic.77759

En la medida en que habitamos cada instante de nuestro presente, el encuentro con el otro se transforma.

Al permanecer atentos, ecuánimes y compasivos nos adentrarnos en el espacio que existe "entre" los dos, y tanto el terapeuta como quien sufre pueden vivir una experiencia sanadora de la que ambos salgan transformados

\section{Introducción}

La intervención más frecuente que realizamos los profesionales sanitarios a lo largo de nuestra vida profesional es la entrevista clínica, o el encuentro terapéutico con nuestros pacientes y sus familiares.

En general, no hemos recibido mucha formación específica para poder afrontar este encuentro con otro ser humano que, junto con su enfermedad, trae consigo su biografía, su manera de percibir el mundo, sus problemas, sus emociones y, que a menudo, viene con sus inquietudes, sus incertidumbres y su sufrimiento.

Nuestra formación académica como profesionales sanitarios, en general se ha basado en un paradigma materialista en el que se da mucha importancia a lo medible, objetivable, cuantificable (signos, síntomas, pruebas diagnósticas, tratamientos) y escaso o nulo valor a nuestra propia subjetividad, a nuestras emociones, y a nuestro mundo interior. De este modo, nos hemos convertido en magos bioquímicos y gigantes tecnológicos, mientras hemos permanecido emocionalmente inmaduros y enanos en sabiduría.

Esta falta de atención a nuestro mundo interior nos ha llevado a no haber explorado ni profundizado en nuestros propios recursos personales. Como terapeutas a menudo desconocemos el potencial de nuestra presencia. Y aunque ha sido reconocida, y existen modelos o propuestas académicas para el cultivo de este enorme recurso que es la presencia terapéutica ${ }^{(1-3)}$, en general en el ámbito sanitario, existe un desconocimiento de estos modelos.

Este artículo pretende profundizar en esta capacidad que todos tenemos, la de estar presentes frente a ese desconocido que sufre y del cual estamos siendo testigos. Se trata de una capacidad que todos podemos reconocer, explorar, cultivar y ponerla al servicio de quienes acompañamos. Quizás solo nos falta algún mapa de este territorio que merece ser más visitado, e indicar un camino que cada uno debe recorrer para poder habitar su propia presencia que, al compartirla, genere mayor profundidad de conexión y eficiencia terapéutica. 
En cuidados paliativos, y especialmente en el acompañamiento del proceso de morir, el encuentro terapéutico en ocasiones puede adquirir mucha intensidad emocional y ser potencialmente impactante para el propio profesional, y a menudo no disponemos de la formación necesaria para brindar una respuesta o tratamientos eficaces en relación con este encuentro íntimo que se desarrolla con el paciente. Sin embargo, disponemos de una herramienta inherente a todo ser humano y accesible para cada encuentro con el otro: "nuestra presencia"; entendida como el contacto con nuestro propio SER, y que nos habilita a conquistar una forma de conectar con el paciente de manera lúcida, serena y creativa.

Esta presencia por parte del terapeuta es la que nos ayuda a realizar un "ajuste creativo" ${ }^{(4)}$ para contrarrestar el impacto, inestabilidad o desequilibrio generado en el encuentro, y recuperar el centro para permanecer en una actitud ecuánime y compasiva frente al desconocido que sufre.

En otras palabras, la presencia nos conecta con un grado de conciencia personal y de aquello que nos rodea, y facilita la estabilidad o equilibrio emocional y la capacidad de regular las emociones que surgen en el encuentro, que a menudo pueden impactar al terapeuta. También, en la medida que nos conecta con la fuente de nuestra paz interior, a pesar de lo caótico que pueda ser el entorno, nos permite realizar los ajustes en el "aquí y ahora" para permanecer en contacto íntimo con el otro. La actitud de presencia nos permite que, aunque los contenidos de sufrimiento del otro nos impacten, podamos reelaborarnos para redefinir ese encuentro in situ y transformar la experiencia en ese instante, para mantenernos más cercanos, y menos dispersos o fragmentados.

Ofrecemos aquí, una actualización sobre que es la presencia, como "actúa" y como se puede promover o cultivar para acercarnos a lo que consideramos esencial del encuentro terapéutico No como una revisión bibliográfica al modo clásico, sino intentando estructurar para el lector interesado la experiencia clínica de los autores: en el entorno de la oncología y los cuidados paliativos.

\section{La relación de ayuda sus riesgos y oportunidades}

Si llevas algunos años como profesional de ayuda, habrás comprobado que nuestra profesión nos permite asomarnos a la intimidad de las vidas de otras personas y con ello a aprender de la vida, de nuestra naturaleza humana y crecer personalmente.

También habrás visto que este trabajo tiene sus riesgos, que no es fácil y que a menudo vives retos para los que sientes que no estas preparado.

Interactuar con personas, pacientes y familiares, en contextos que requieren grados elevados de compromiso personal, obliga a gestionar de manera adecuada la implicación de forma que no sea excesiva, lo que aumentaría la identificación, o demasiado desapegada (evitativa), lo que comportaría despersonalización y la posible aparición de procesos de auto culpabilización. Fluir entre estos dos polos, habitando el presente y gestionando el grado adecuado de implicación no se nos enseña en las facultades y debemos aprenderlo de forma autodidacta.

Cuando los profesionales sanitarios estamos expuestos al contagio/saturación emocional, y sin recursos para atender el sufrimiento, hay un riesgo de acabar por padecer el desgaste por empatía (mal llamado fatiga de compasión) y de adoptar actitudes de desprotección personal o conductas evitativas como el cinismo o el desinterés por los pacientes, que finalmente conducen al burnout. 
Nuestro trabajo puede saturarnos, agotarnos, agobiarnos y hasta producir problemas de salud mental; y también pude ser una fuente de satisfacción, y una oportunidad de aprendizaje y de crecimiento personal. El quid de esta diferencia es lo que intentamos explorar en este texto.

Ni huir ni contagiarse, sino abrirnos paso a una presencia segura, que genere confianza:

Como hemos comentado, el sistema sanitario y la formación académica de los profesionales sanitarios, -en general-, aún no ha integrado la dimensión subjetiva, la vivencia del paciente y el cortejo emocional que surge ante el sufrimiento, el deterioro, la vulnerabilidad y la dependencia que caracterizan la enfermedad terminal, como parte de nuestra formación profesional.

Tampoco hemos entendido ni atendido como merecen los aspectos dinámicos psicológicos e incluso fisiológicos, que se dan en el encuentro terapéutico y que nos permiten gestionar de forma constructiva la relación terapéutica.

Ante la falta de formación y de recursos personales para entender y acompañar el sufrimiento gestionando las emociones que surgen en el proceso, los profesionales a veces nos debatimos entre dos acciones: huir de lo que sentimos amenazante o luchar para no sobre implicarnos y contagiarnos, sin embargo, ambas actitudes tienen consecuencias negativas.

La sobre implicación acaba frecuentemente con lo que se ha llamado desgaste por empatía (o fatiga por compasión), y la huida suele ser preámbulo de despersonalización y perdida de sentido profesional.

En la medida que aprendemos a entender nuestro papel y a gestionarlo de forma constructiva vamos descubriendo el potencial sanador que subyace, tanto para el paciente como para el terapeuta, en la relación de ayuda.

La diferencia entre: protegerse y huir, o luchar y sufrir; o entender, atender y crecer en la relación en el encuentro con el que sufre, reside básicamente en la calidad de la atención y la presencia que el terapeuta aporta a la relación.

\section{Adentrándonos en la fisiología de las emociones y su gestión}

La emoción tal como la definen Eckman $^{(5)}$, Levenson $^{(6)}$ y $\operatorname{Gross}^{(7)}$ es un proceso caracterizado por su activación automática influenciada por nuestro pasado evolutivo y personal, a través del cual percibimos que ocurre algo importante para nuestro bienestar, y se asocia a una serie de cambios fisiológicos y conductas orientados a afrontar esta situación. Se supone que aparecen para ayudarnos a coordinar una respuesta eficiente, ayudándonos a afrontar importantes retos y oportunidades.

Las emociones se caracterizan por ser señales universales específicas, con una fisiología propia, surgen automáticamente cuando se desencadena su detonador, muchos de ellos con características universales. Se perciben en el cuerpo, aunque en ocasiones pueden ser inconscientes. Aparecen rápidamente, involuntariamente como una experiencia subjetiva que surge ante situaciones reales o imaginadas, y que son relevantes para la persona y nos empujan a responder a movernos en algún sentido y actuar.

Tienen una función interesante, que resumidamente sería informarnos de la oportunidad o amenaza de la situación que se vive. Se perciben en el cuerpo, se 
expresan en la cara, en el lenguaje corporal, el tono de voz, la prosodia, y se pueden percibir en las caras, los cuerpos y la voz de los demás, de modo que son una forma de comunicación con los otros o de conexión social. Es decir, las emociones nos conectan y nos informan de la vivencia interna propia y del otro.

El problema surge cuando la emoción, debido a su intensidad o falta de regulación por parte de quien la percibe, inunda la conciencia, "toma el mando" y se instala en el centro de la atención transformándose en la protagonista de las reacciones que se suceden de forma automática, sin equilibrio, y que conducen a actuar de forma reactiva.

El equilibro emocional se da cuando la persona es consciente de la emoción (la percibe en su cuerpo, toma conciencia de esta) y de la "información" que nos aporta, y que tras regular el impulso es capaz de aprovechar la energía que esta mueve internamente para orientarla hacia una respuesta constructiva.

El concepto de inteligencia emocional se ha descrito por Solovey y Mayer en $1990^{(8)}$ como: "la capacidad de percibir las emociones, acceder y generarlas para ayudar al pensamiento, comprender las emociones y los significados emocionales, y regular reflexivamente las emociones para promover un mejor crecimiento emocional e intelectual".

Pero para esto hace falta que la persona este muy bien conectada con sus propias sensaciones corporales (interocepción) y disponga de suficiente nivel de autoconciencia para aprovechar el pequeño espacio de microsegundos que se da entre el detonante y la respuesta. Un espacio que Paul Eckman metafóricamente llama "la chispa antes de la llama", entre la chispa y la llama la persona altamente consciente es capaz de responder constructivamente en lugar de reaccionar automáticamente. Esto según Víctor Frankl es nuestro espacio de libertad: "Entre el estímulo y la respuesta hay un espacio, en este espacio reside nuestro poder para elegir la respuesta. En nuestra respuesta reside nuestra madurez y nuestra libertad".

El grado de autoconciencia del profesional, su grado de conexión consigo mismo, es lo que le permite percibir y elaborar sus emociones antes de reaccionar, y a su vez, es lo que le ayuda a elaborar una respuesta constructiva aprovechando e integrando la energía propia de cada emoción. Este equilibrio que surge de sentir, conectar, entender y atender a las emociones en uno mismo; y de crear la oportunidad de coregulación con el paciente lo que se necesita para poder actuar en nuestro entorno clínico con sabiduría y compasión, sin sobre implicarse ni huir de la aparente amenaza del sufrimiento ajeno.

\section{La presencia como herramienta en la práctica clínica: el poder terapéutico de habitar el presente}

En algunas publicaciones previas hemos hablado de: hospitalidad, presencia y compasión como herramientas de acompañamiento espiritual ${ }^{(9,10)}$ en cuidados paliativos y también hemos evidenciado la importancia de la autoconciencia del profesional de salud como elemento crucial en la protección del desgaste profesional ${ }^{(11)}$.

Hoy podemos afirmar que la auto conciencia es la plataforma inicial sobre la cual se promueve y constituye la actitud de presencia, en donde el terapeuta puede sostenerse y magnificar su experiencia de confianza, compasión, sabiduría y coraje; habilidades que le permiten profundizar el encuentro terapéutico, y que condicionan en gran manera los posibles resultados de este encuentro. La actitud de presencia 
nos ayuda a realizar un ajuste creativo en el aquí y ahora, nos habilita a responder asertivamente a las variables que se hacen manifiestas en cada encuentro en particular, con cada persona que atendemos.

Desde hace años, se ha reconocido al profesional como la herramienta, que desde su propia presencia participa e interviene en el proceso terapéutico. Sabemos que el calibrado de esta herramienta es de crucial importancia en el mantenimiento de su capacidad para trabajar de forma eficiente, disfrutando del ejercicio de su vocación y aprendiendo en cada encuentro ${ }^{(12)}$

Cicely Saunders nos recordaba en su libro "Velad Conmigo"(13), que cuando los enfermos viven una experiencia de sanación te dejan un legado, una herencia. Es decir, una vivencia que nos cambia la manera de ver, nos genera cada vez mayor confianza, y que el profesional puede llevar luego a la cabecera de los enfermos que verá después.

Hay un efecto sobre el terapeuta de cada intervención en la que está presente y vive de forma creativa. Cada encuentro produce un impacto que no le deja indiferente. Hoy sabemos que cuando el enfermo atraviesa su malestar y accede a niveles de conciencia que le permiten integrar la experiencia dolorosa se produce un crecimiento, lo que denominamos crecimiento post traumático o sanación, entendida como la recuperación de la integridad. Y el terapeuta que comparte la experiencia suele vivir también un crecimiento en este caso llamado "crecimiento vicario" o secundario"(14). Desde esta perspectiva trabajar como terapeuta con presencia es una de las mejores experiencias para el despliegue de nuestra propia espiritualidad.

\section{5. ¿Qué es la presencia terapéutica?}

Significa que el terapeuta lleva todo su ser al encuentro y que habita completamente el momento presente en múltiples niveles: físico, emocional, cognitivo, relacional y espiritual.

Supone estar enraizado en la profundidad de uno mismo y al mismo tiempo con el lugar que habita y conectado al paciente, prestando especial atención a la expresión verbal y no verbal de la experiencia del paciente.

Implica estar abierto a vivenciar lo que es, ser testigos de lo que surja en la realidad del encuentro. Mientras, vamos tomando consciencia de nuestras tendencias y patrones habituales de elaboración e interpretación, y esto nos habilita a desplegar nuevas formas de responder.

Incluye que el terapeuta vivencie una experiencia interna de sentirse:

1) En contacto consigo mismo, enraizado en su propia profundidad, en su esencia que es fuente de serenidad, de paz, de seguridad, con confianza y sin miedo.

2) Al mismo tiempo está conectado con el sufrimiento de la persona que atiende sin huir, acogiendo todo lo que se da en el encuentro y

3) Y siendo capaz de percibir una perspectiva más amplia y espaciosa, lo que se llama en "expansión" viendo más allá de lo inmediato y con confianza en el proceso que va a seguir desplegándose $\mathrm{y}$

4) Sintiéndose conectado con y al servicio del otro, comprometido con la sanación del paciente.

En conclusión, la presencia, es una forma de estar con el paciente que optimiza la relación terapéutica ${ }^{(15)}$. Nos permite fluir, mantenernos estables, abiertos, atentos y 
disponibles frente a cualquier cosa que surja en el encuentro; es lo que nos permite abrazar la experiencia del paciente y la nuestra, pasando de una experiencia contraída de la realidad a una experiencia magnificada.

Y es el reconocimiento de nuestro mundo interno, de la interconexión y de la coregulación lo que hace de la presencia, el fundamento de la calidad de la intervención terapéutica. Como dice Michael Kearney: "La vida interior del clínico determina la calidad de la presencia que aporta a la cabecera de la cama del enfermo" (16)

Algunas descripciones de autores referentes sobre la presencia

Carl Rogers ${ }^{(2)}$ en sus últimos escritos afirmó la importancia de la presencia:

"Cuando estoy en mi mejor momento, como facilitador de grupo o como terapeuta, descubro otra característica. Encuentro que cuando estoy más cerca de mi ser interno e intuitivo, cuando estoy de alguna manera en contacto con lo desconocido en mí, cuando tal vez estoy en un estado de conciencia ligeramente alterado, entonces cualquier cosa que haga parece estar llena de sanación. Entonces, simplemente mi presencia es liberadora y útil para el otro. No hay nada que pueda hacer para forzar esta experiencia, pero cuando puedo relajarme y estar cerca del núcleo trascendental de mí, entonces puedo comportarme de manera extraña e impulsiva en la relación, formas en las que no puedo justificarme racionalmente, que no tienen nada que ver con mis procesos de pensamiento. Pero estos comportamientos extraños resultan ser correctos, de alguna manera extraña: parece que mi espiritu se ha extendido y ha tocado el espíritu del otro. Nuestra relación se trasciende y se convierte en parte de algo más grande. El crecimiento profundo y la sanación y la energía están presentes"

Yalom $^{(17)}$ nos recuerda que:

La presencia separada le permite al terapeuta estar "en dos lugares a la vez: a su lado y al lado del paciente". "Los terapeutas efectivos. . saben mostrar una calidez no posesiva y un alto grado de empatía precisa y son capaces de "Estar con" o "comprender el significado" de un paciente ". "El terapeuta está interesado en el" usted" del paciente, no solo en el" usted "que está presente, sino en el potencial" el usted "latente". En este encuentro auténtico, el terapeuta anhela el "estar con", sin prejuicios.

Castillejo $^{(18)}$ afirma que la presencia se despliega en el encuentro: "el terapeuta se relaciona con el paciente de una manera genuina y solidaria y se esfuerza por lograr momentos de autenticidad del encuentro "... " Este encuentro es algo asi como un milagro, y no se puede planificar ni explicar". Es como si "un misterioso" tercero "estuviera presente. Tales reuniones "nos suceden como una gracia" y no pueden ser forzadas.

Du Mont ${ }^{(19)}$ describe los atributos de la presencia como:
(a) proximidad, estar allí;
(b) disponibilidad;
(c) autenticidad;
(d) reciprocidad / mutualidad;
(e) compromiso / conexión; 
(f) significado compartido; y

(g) acciones del terapeuta que "reflejan la creencia de que el paciente tiene dentro de sí una forma única de ser"

Otro autor, Stanley ${ }^{(20)}$ habla de la presencia como un modo de ser, que requiere:

(a) conocer y sentirse cómodo consigo mismo,

(b) conocer a la otra persona,

(c) conexión,

(d) afirmación y valoración,

(e) reconocimiento de vulnerabilidad,

(f) intuición,

(g) empatía y la voluntad de ser vulnerable,

(h) estar en el momento,

(i) serenidad y silencio, y

(j) que puede ser trascendente.

La presencia sanadora (desde la perspectiva de los curanderos tradicionales) ${ }^{(21)}$ consiste principalmente en (a) el amor y (b) la buena intención. Los componentes adicionales incluyen, pero no están limitados a, gracia espiritual, creencia y conciencia enfocada.

Otros autores ${ }^{(22)}$ se refieren a la presencia sanadora como "un fenómeno interpersonal, intrapersonal y transpersonal hacia la trascendencia que conduce a un cambio espiritual beneficioso, terapéutico y / o positivo dentro del otro individuo... y ...dentro del sanador"

Geller y Porges ${ }^{(23)}$ nos dicen que "La presencia terapéutica comienza con el terapeuta cultivando la presencia antes de una sesión y conociendo al paciente desde este estado de presencia. Informan que la experiencia de la presencia terapéutica implica concurrentemente:

(a) estar enraizados y en contacto con uno mismo de forma íntegra y saludable;

(b) estar abierto, receptivo e inmerso en lo conmovedor del momento;

(c) tener una mayor sensación de amplitud y expansión de la conciencia y la percepción.

Esta conciencia fundamentada, inmersa y expandida también ocurre con

(d) la intención de estar con y para el cliente en el servicio de su proceso de sanación.

Al estar conectado a la tierra (enraizado), inmerso y espacioso, con la intención de estar con y para el otro, el terapeuta invita al cliente a un estado más profundo y compartido de presencia terapéutica relacional."

Naranjo ${ }^{(24)}$ define la presencia como una disposición a vivenciar en el presente nuestra experiencia interna, cualquiera que sea, en contacto con la realidad externa de manera simultánea, tomando consciencia, con atención plena a cada vivencia y percatándonos de nosotros mismos. Propone "darnos cuenta" en el "aquí y ahora" de cómo nos sentimos, de cómo pensamos, y de cómo nos experimentamos a nosotros mismos, al otro y al entorno en el que se desarrolla la experiencia. Invitándonos a 
estar receptivos y abiertos a vivenciar en el presente la experiencia tal como es, más allá de nuestras interpretaciones, para poder detectar y hacer contacto con lo que necesitamos en ese momento dado.

\section{6. ¿Cuál es la base neurofisiológica de la presencia terapéutica?}

La teoría Polivagal de Stephen Porges ${ }^{(25)}$ junto con sus aplicaciones clínicas ${ }^{(26)}$ nos permiten integrar "el sistema de conexión social" que hemos desarrollado los mamíferos y más específicamente los humanos. La conexión que se establece en el encuentro terapéutico se sostiene sobre circuitos orgánicos específicos (sistema nervioso autónomo, neurocepción, y sistemas de protección/defensa, desarrollados filogénicamente), cada vez mejor comprendidos.

Sabemos que estamos dotados de un sistema de "escaneo" constante que explora las señales que recibimos del entorno en búsqueda de amenazas y oportunidades, y son captadas por nuestro sistema fisiológico (neurocepción).

A través de la neurocepción recibimos información sub cortical (inicialmente no consciente) que se transmite a través del sistema neurovegetativo, y por vía aferente a través del neumogástrico hacia el SNC. Cuando la información es relevante y se hace consciente hablamos de interocepción (la intuición que nos ofrece información de algo que nos interesa o conviene para nuestra supervivencia).

En la interconexión social hemos desarrollado formas para avisarnos del potencial riesgo en el entorno, o de que nos encontramos en un ambiente de seguridad.

La transmisión de esta información se da a través de las micro expresiones faciales (tono relajado o tenso de la parte superior de los músculos de la cara), el lenguaje corporal de tensión /relajación, cierre / apertura, y también por el tono de voz, el timbre, la calidez y la prosodia.

Nos conectamos a través de los mensajes que nos mandamos mutuamente, y este sistema de conexión social es la plataforma de conexión entre el paciente y terapeuta.

En la medida que el paciente se siente amenazado por la enfermedad, su sistema vegetativo está en modo amenaza (lucha, huida o parálisis).

Desde esta perspectiva, el encuentro terapéutico puede ser visto como una oportunidad. Sabemos que cuando el terapeuta esta enraizado en su "presencia" es capaz de trasmitir al paciente seguridad y confianza, y que en la medida que el paciente se conecta y resuena con la actitud del terapeuta puede ir apagando/ modulando su estado de alarma, y a través de la co-regulación que se establece, abrirse a la posibilidad de pasar del estado de amenaza, peligro y tensión, a niveles de serenidad, confianza y paz que le permitan el acceso a sus propios recursos internos, que en el estado de amenaza no percibía.

La presencia es altamente relacional, la serenidad, confianza y paz interior que aporta el terapeuta al encuentro es percibida por el paciente que, al sentirse escuchado, percibido, entendido, y no juzgado, va naturalmente conquistando una experiencia de confianza; y poco a poco, a medida que su sistema nervioso se adapta a la presencia tranquilizadora del terapeuta, se va calmando. Las defensas del paciente se suavizan, de modo que el crecimiento natural y la sanación se pueden desplegar.

A medida que ambos se conectan, el terapeuta percibe la experiencia del paciente y también es impactado por ella, y manteniéndose en el presente es capaz de elaborar 
su propia emocionalidad, de reconocer su propio diálogo interno y de descubrir qué es lo que se interpone entre él y la posibilidad de estar realmente presente para el otro, para poder responder de forma más adecuada a las necesidades suyas y a las del paciente en función de lo que está sucediendo en ese momento.

La presencia está relacionada con la empatía y la compasión, pero es diferente y previa a ambas. Es la plataforma desde la cual tomamos consciencia de nuestros ruidos internos, de aquel pasado que se hace presente en resonancia con nuestro paciente, o de nuestras ensoñaciones proyectadas a futuro; y desde la cual podemos permitirnos realizar un ajuste creativo para responder de manera más eficiente a las necesidades que se hacen manifiestas en el aquí y ahora, y que permite profundizar el lazo de confianza con nuestro paciente, que al sentirse "sentido" por nosotros, será capaz de permitirse fluir y profundizar en su dolor y su pena.

\section{7. ¿Cuáles son las cualidades características de la presencia?}

Reconocemos cuatro características principales: Apertura / Claridad / Ecuanimidad / Vitalidad.

La primera cualidad es la apertura, nos referimos a lo que se conoce como conciencia abierta para observar y percibir lo que se encuentra frente a nosotros con una forma progresiva de disponibilidad que nos permite percibirlo todo sin apropiarnos ni juzgar nada. Es un estado de conciencia que surge de la confianza y de la compasión, que trasmite serenidad y paz interior a los que perciben este estado mental, y que genera seguridad y confianza. La apertura llama a la apertura y el paciente que se siente apreciado desde esta cualidad, puede suavizar sus resistencias y disponerse o abrirse más fácilmente al encuentro.

Otra cualidad es la claridad, esta se compone de lucidez y luminosidad, y surge del silencio de la mente. La conciencia liberada de pensamiento tiene su propia inteligencia y sabiduría. Desde la claridad de una mente serena, se puede percibir la realidad sin condicionamientos y también acceder a intuiciones sobre las dificultades del enfermo y sus recursos, esto magnifica la experiencia del encuentro y nos permite acceder a una mayor capacidad de entender, atender y acompañar. Esta claridad también puede ser beneficiosa para el paciente, que estando en contacto con esta cualidad podrá entender mejor sus dificultades y ocasionalmente encontrar él mismo los recursos para afrontarlas. Podríamos resumir lo expuesto anteriormente en que: "cuando tú me escuchas... yo me entiendo".

Otra cualidad es la ecuanimidad, caracterizada por la posibilidad de permanecer en un estado de equilibrio y compostura emocional serena que no se ve perturbada a pesar de la intensidad de las emociones que surgen en el encuentro. Esta característica incluye tres cualidades: imparcialidad, estabilidad y equilibrio, que son las que nos permiten fluir frente a los acontecimientos internos y externos sin juzgarlos ni oponer resistencia. Esta actitud ecuánime, se transmite también al otro y ayuda a modular "la tormenta" que el enfermo pueda traer consigo, como decía Marco Aurelio, un filósofo estoico hace casi dos mil años "Debes ser como un promontorio en el mar, contra el cual las olas baten continuamente, pero resiste, y en su cercanía las crecidas olas se tranquilizan y aquietan".

La cuarta cualidad es la vitalidad, que implica la posibilidad de habitar el momento de forma enérgica o vívida, y que proporciona la sensación de estar plenamente vivo, 
junto con una gran fuerza interior, y normalmente se asocia con una sensación de alegría y de gozo. Esta vitalidad permite mantener el contacto con el paciente con un nivel de atención y calidez que habilita que surja la creatividad en el encuentro, lo nuevo, lo inesperado. El enfermo que sufre y se siente desvitalizado, puede percibir y acogerse también a la energía que se da en el encuentro.

\section{Co-regulación: la presencia en acción}

¿Cómo se desarrolla lo que llamamos la co-regulación en la relación terapéutica? Geller y Porges ${ }^{(23)}$ afirman que:

"Hay relaciones recíprocas entre la presencia sentida y comunicada del terapeuta y la de los pacientes. Esta presencia que crece dentro y entre el terapeuta y los pacientes contribuye al desarrollo de la presencia relacional. La presencia relacional proporciona las condiciones para un encuentro "Yo-Tú" y, en última instancia, esta presencia relacional mutua también promueve la profundidad relacional, la seguridad y el cambio terapéutico". Esta experiencia Yo-Tu ha sido bellamente descrita también por Martin Buber ${ }^{(27)}$.

Se ha definido co-regulación como el vínculo bidireccional de las emociones oscilantes entre diferentes participantes, contribuyendo a la estabilidad emocional de ambos: es decir, la co-regulación implica la regulación mutua del estado fisiológico entre las personas ${ }^{(23)}$. Por ejemplo, en la diada madre-bebé, cuando el bebé llora, no solo la madre calma al pequeño, sino que la respuesta del niño que se relaja y tranquiliza con los sonidos, las expresiones faciales y los gestos de la madre, tienen el efecto recíproco de calmarla a ella.

Cuando el terapeuta se encuentra tranquilo, emocionalmente equilibrado y enraizado en su SER, es capaz de ofrecer una actitud de presencia plena que va a poder ser percibida y asumida por el paciente mediante la co-regulación.

Desde esta perspectiva se entiende la necesidad de que el profesional de la salud cultive su presencia terapéutica para poder aportarla en el encuentro con el paciente en su máximo potencial.

\section{9. ¿Cómo se puede cultivar la presencia?}

Hay muchos caminos para desarrollar nuestra capacidad de mantenernos presentes. El primer paso consiste en reconocer que nuestro nivel de conciencia, nuestra capacidad de estar presentes, atentos, de forma lucida, estable y relajada, se puede cultivar. Por lo tanto, el grado de presencia que tenemos siempre es mejorable.

Como cualquier habilidad que queramos promover requiere de una práctica regular. Los atisbos de presencia nos conducirán a profundizar en sus cualidades, con el tiempo, la presencia terapéutica se transformará en una herramienta a la que podremos acceder en forma voluntaria, para luego transformarse en una actitud.

Al tratarse de una capacidad inherente de todo ser humano la presencia la podemos promover a través de: 
- La práctica regular del cultivo de nuestra atención: meditación, oración contemplativa, mindfulness, la actividad artística creativa, la contemplación de la naturaleza y de la belleza.

- La experiencia acumulada durante nuestra práctica clínica habitual en actitud de presencia: la conciencia de nosotros mismos y el saber del otro.

- Espacios de reflexión y supervisión psicoterapéutica individual y en equipo de nuestra práctica clínica, en donde podamos elaborar nuestras formas de hacer contacto con el paciente y todo lo que se suscita dentro del encuentro terapéutico.

- Formación y práctica de modelos teóricos que contribuyan al desarrollo de la presencia.

Shari M. Geller ha publicado "Una guía práctica de cómo cultivar la presencia"(28). En ella, nos invita a tomarnos un momento para hacer una pausa, esto nos ayuda a reducir la velocidad y crear un espacio en torno a la experiencia de modo que podamos notar lo que estamos experimentando o lo que bloquea nuestra capacidad de estar completamente aquí con nosotros mismos y/o con otra persona. A permitirnos abrir nuestros sentidos: ver, escuchar, oler todos los intrincados detalles de lo que nos rodea. Y a respirar profunda y lentamente. Tomar respiraciones más largas y más lentas nos permite calmar el sistema nervioso simpático y activar el tono vagal. Por último, recomienda establecer una intención de presencia. Se recomienda iniciar la práctica para cultivar la presencia antes de iniciar la sesión terapéutica.

\section{Reflexiones finales a modo de conclusión}

La presencia, es una forma de vivir, de estar con nosotros y con el paciente que optimiza la relación terapéutica, y que cultiva una conexión relacional segura y de apoyo. Significa que el terapeuta lleva todo su ser al encuentro y que habita completamente el momento presente en múltiples niveles: físico, emocional, cognitivo, relacional y espiritual. Supone estar enraizado en la profundidad de uno mismo, y al mismo tiempo nos habilita a percatarnos de cómo nos sentimos, de cómo pensamos, y de cómo nos experimentamos a nosotros mismos, al otro y al entorno en el que se desarrolla la experiencia. Implica estar abierto a vivenciar lo que es. Y nos permite pasar de una experiencia contraída de la realidad a una experiencia magnificada.

Reconocemos cuatro cualidades características principales: Apertura, Claridad, Ecuanimidad y Vitalidad. A nivel relacional, la serenidad, confianza y paz interior que aporta el terapeuta en actitud de presencia es percibida por el paciente que, al sentirse escuchado, percibido, entendido y no juzgado, va naturalmente conquistando una experiencia de seguridad y confianza. Esta presencia relacional mutua también promueve la profundidad relacional, la seguridad y el proceso terapéutico de ambos.

Nuestro nivel de conciencia, nuestra capacidad de estar presentes, atentos, de forma lucida, estable y relajada, se puede cultivar. Por lo tanto, el grado de presencia que tenemos siempre es mejorable.

El autocuidado de los profesionales de la salud basado en la autoconciencia ${ }^{(29)}$ es la plataforma inicial sobre la cual se promueve y constituye la actitud de presencia, en donde el terapeuta puede sostenerse y magnificar su experiencia de confianza, compasión, sabiduría y coraje; habilidades que le permiten profundizar el encuentro terapéutico. Supone: profundizar en sí mismo, trabajar conscientemente, acceder a niveles elevados 
de conciencia, para pasar de sentirse amenazado por el sufrimiento del otro, a sentirse agradecido de poder aportar su forma de estar, de ser y de vivenciarse como una herramienta para que el otro también pueda conectar con su propia profundidad. Una profundidad que no pertenece a ninguno y es la plataforma del ser de ambos.

La práctica regular del cultivo de nuestra atención como la meditación, oración contemplativa, mindfulness, la actividad artística creativa, y la contemplación de la naturaleza y la belleza, junto a los espacios de reflexión y supervisión de nuestra práctica clínica y a la capacitación en modelos teóricos que contribuyan al desarrollo de la presencia constituyen algunos de los caminos principales para el desarrollo de nuestra presencia terapéutica. Esto implica no solo la práctica, sino una disposición e intención de conquistar una experiencia viva, directa, profunda e inmediata de la realidad ${ }^{(30)}$.

En definitiva, la presencia del terapeuta surge del grado de conciencia que haya desarrollado de sí mismo, de la conexión que haya logrado establecer con su SER, con la profundidad que le sostiene y con aquello que lo rodea. Viviendo nuestro trabajo de esta manera, acompañar a los pacientes se constituye en uno de los caminos para el propio desarrollo espiritual.

En la medida en que trabajamos desde una conciencia despierta, presente, ecuánime y compasiva, podremos establecer relaciones sanadoras para los pacientes y vivenciar experiencias trasformadoras para ambos.

...Entonces, habitar el momento presente se convierte en una forma de ser, estar, experimentar y vivir gozosamente.

\section{Agradecimientos}

AAssumpta Mateu Domènech, amiga y maestra, por su revisión crítica y constructiva al texto.

\section{Referencias bibliográficas}

1. Lewin K. La teoría del campo en la ciencia social. Barcelona: Paidós,1988.

2. Rogers CR. A way of being. Houghton Mifflin, 1980.

3. Perls F, Hefferline RF, Goodman P. Terapia Gestalt: Excitación y crecimiento de la personalidad. Madrid, España: Soc. de cultura Valle-Inclan, 2002

4. Perls F, Hefferline RF, Goodman P. El ajuste Creativo. En: Terapia Gestalt: Excitación y crecimiento de la personalidad. Madrid, España: Soc. de cultura Valle-Inclan,2002

5. Ekman P. An argument for basic emotions. Cogn Emot 1992;6:169-200. https://dx.doi.org/10.1080/02699939208411068

6. Averill JR, Clore GL, Frijda NH, Levenson RW, Scherer KR, Clark LA, et al. What is the function of emotions? In: Ekman P, Davidson RJ, editors. Oxford University Press; 1994. p. $97-177$.

7. Gross JJ. Emotion regulation: Past, present, future. Cogn Emot 1999;13:551-73. https:// dx.doi.org/10.1080/026999399379186 
8. Salovey P, Mayer JD. Emotional intelligence. Imagin Cogn Pers 1990;9:185-211. https:// dx.doi.org/10.2190/dugg-p24e-52wk-6cdg

9. Benito E, Barbero J, Dones M. Espiritualidad en Clínica. Monografías SECPAL; 2014. [Acceso 1 de abril de 2021]. Disponible en: http://www.secpal.com//Documentos/Blog/ Monografia\%20secpal.pdf

10. Benito E, Dones M, Babero J. El acompañamiento espiritual en cuidados paliativos. Psicooncología 2016; 13, 367-84. https://dx.doi.org/10.5209/psic.54442

11. Sansó N, Galiana L, Oliver A, Pascual A, Sinclair S, Benito E. Palliative Care professionals' inner life: Exploring the relationships among awareness, self-care, and compassion satisfaction and fatigue, burnout, and coping with death. J Pain Symptom Manage 2015; 50: 200-7. https://dx.doi.org/10.1016/j.jpainsymman.2015.02.013

12. Novack DH, Suchman AL, Clark W, Epstein RM, Najberg E, Kaplan C. Calibrating the physician. Personal awareness and effective patient care. Working Group on Promoting Physician Personal Awareness, American Academy on Physician and Patient. JAMA 1997;278:502-9. https://dx.doi.org/10.1001/jama.278.6.502

13. Saunders C. Velad Conmigo. Inspiración para una vida en cuidados paliativos. IAHPC Press, 2011.

14. Harrison RL, Westwood MJ. Preventing vicarious traumatization of mental health therapists: Identifying protective practices. Psychotherapy 2009;46:203-19. https:// dx.doi.org/10.1037/a0016081

15. Porges S. Guía de Bolsillo de la Teoría Polivagal. El poder transformador de sentirse seguro. Barcelona: Elefhteria, 2018.

16. Kearney M, Weininger R. Whole person self-care: Self-care from the inside out. In: Whole Person Care. New York, NY: Springer New York, 2011. p. 109-25. https://dx.doi. org/10.1007/978-1-4419-9440-0_10

17. Yalom ID. Existential Psychotherapy. London, England: Basic Books, 1980.

18. De Castillejo IC. Knowing Woman. Boston, MA: Shambhala Publications, 1990.

19. Du Mont PMD. The concept of therapeutic presence in nursing [Internet]; 2006 [Acceso el 29 de junio de 2021]. Disponible en: http://citeseerx.ist.psu.edu/viewdoc/download?do $\mathrm{i}=10.1 .1 .530 .6075 \& \mathrm{rep}=\mathrm{rep} 1 \&$ type $=\mathrm{pdf}$

20. Stanley KJ. The healing power of presence: respite from the fear of abandonment. Oncol Nurs Forum 2002;29:935-40. https://dx.doi.org/10.1188/02.ONF.935-940

21. Jonas WB, Crawford CC. The healing presence: can it be reliably measured? J Altern Complement Med 2004;10:751-6. https://dx.doi.org/10.1089/acm.2004.10.751

22. McDonough-Means SI, Kreitzer MJ, Bell IR. Fostering a healing presence and investigating its mediators. J Altern Complement Med 2004;10 Suppl 1:S25-41. https:// dx.doi.org/10.1089/1075553042245890

23. Geller SM, Porges SW. Therapeutic presence: Neurophysiological mechanisms mediating feeling safe in therapeutic relationships. J Psychother Integr 2014;24:178-92. https:// dx.doi.org/10.1037/a0037511

24. Naranjo C. El centrarse en el presente como técnica, prescripción e ideal. En: La vieja y novísima Gestalt: actitud y práctica de un experiencialismo ateórico. Santiago de Chile: Cuatro Vientos, 2011.

25. Porges SW. The polyvagal theory: Neurophysiological foundations of emotions, attachment, communication, and self-regulation. New York, NY: WW Norton, 2011.

26. Geller S. Presencia terapéutica y teoría polivagal: principios y prácticas para cultivar las relaciones terapéuticas efectivas. En: Porges SW, Deb D, editores. Aplicaciones clínicas de la Teoría Polivagal. Barcelona, España: Eleftheria; 2019.p. 151-176 
27. Buber M. Yo y tú. Barcelona: Herder; 2017

28. Geller S. Tips for cultivating presence [Internet]. Sharigeller.ca. [Acceso el 29 de junio de 2021]. Disponible en: https://www.sharigeller.ca/cultivating_presence_tips.php

29. Benito E, Rivera PR. El cultivo de la autoconciencia y el bienestar emocional en los profesionales que trabajan con el sufrimiento: Autoconciencia y Bienestar Emocional. Revista de Investigación y Educación en Ciencias de la Salud (RIECS). 2019;4(S1):7793. https://dx.doi.org/10.37536/riecs.2019.4.s1.115

30. Cavallé M. El camino de la presencia [Internet]. YouTube; 2020 [Acceso el 29 de junio de 2021]. Disponible en: https://www.youtube.com/watch?v=gudF4yRi1qQ 